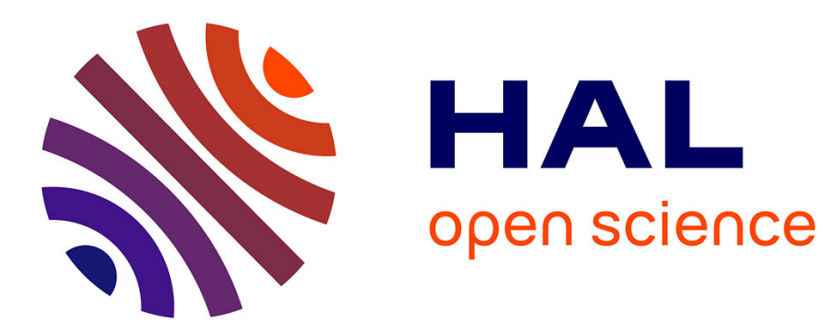

\title{
Couple électromécanique des machines à réluctance Vernier excitées à commutation électronique
}

\author{
G. Drouet, J. Faucher, L. Banon
}

\section{To cite this version:}

G. Drouet, J. Faucher, L. Banon. Couple électromécanique des machines à réluctance Vernier excitées à commutation électronique. Revue de Physique Appliquée, 1984, 19 (3), pp.245-251. 10.1051/rphysap:01984001903024500 . jpa-00245190

\section{HAL Id: jpa-00245190 https://hal.science/jpa-00245190}

Submitted on 1 Jan 1984

HAL is a multi-disciplinary open access archive for the deposit and dissemination of scientific research documents, whether they are published or not. The documents may come from teaching and research institutions in France or abroad, or from public or private research centers.
L'archive ouverte pluridisciplinaire HAL, est destinée au dépôt et à la diffusion de documents scientifiques de niveau recherche, publiés ou non, émanant des établissements d'enseignement et de recherche français ou étrangers, des laboratoires publics ou privés. 


\title{
Couple électromécanique des machines à réluctance Vernier excitées à commutation électronique
}

\author{
G. Drouet, J. Faucher et L. Banon \\ Laboratoire d'Electrotechnique et d'Electronique Industrielle $\left(^{*}\right), 2$, rue Camichel, 31071 Toulouse Cedex, France
}

(Reçu le 16 juin 1983, révisé le 5 décembre, accepté le 8 décembre 1983)

\begin{abstract}
Résumé. - Les auteurs présentent une méthode d'étude des machines à réluctance Vernier excitées par courant statorique et alimentées par commutateur de courant. L'utilisatión du développement en série de Fourier permet de définir les structures générales fonctionnant sur les termes fondamentaux des grandeurs électriques et magnétiques. Une étude de la stabilité en couple est effectuée dans un premier temps par méthode analytique simplifiée, dans un second temps par simulation de l'ensemble machine convertisseur basée sur la méthode des circuits magnétiques équivalents et du calcul local de champ.
\end{abstract}

\begin{abstract}
The authors present an analysis of torque characteristics for current source converter fed reluctance machines including a stator field winding. A Fourier series analysis of electric and magnetic variables allows to define three different kinds of magnetic structures only by considering the influence of the first harmonic. The study of torque stability is then conducted by using a simplified analytical method. In a last part, results are determined from a complete simulation of overall machine converter drive by using equivalent magnetic circuits and local magnetic field computation.
\end{abstract}

\section{Liste des principaux symboles.}

$\alpha \quad$ position angulaire d'un point de l'entrefer par rapport au stator $\left(\mathrm{rad} . \mathrm{ou}^{\circ}\right)$.

$B \quad$ induction magnétique (T).

$C$ couple électromagnétique (N.m).

$\delta_{\text {is }} \quad$ densité linéique de courant statorique le long de l'entrefer $(\mathrm{A} / \mathrm{m})$.

$\varepsilon \quad$ longueur d'entrefer $(\mathrm{m})$.

$l_{0} \quad$ longueur de circuit magnétique $(\mathrm{m})$.

$N_{\mathrm{s}} \quad$ nombre de dents statoriques.

$N_{\mathrm{r}}$ nombre de dents rotoriques.

$\mathfrak{T}_{\theta}(\alpha)$ fonction densité de perméance (T/A).

$\mathfrak{J}_{0} \quad$ composante continue de $\mathfrak{T}_{\theta}(\alpha)(\mathrm{T} / \mathrm{A})$.

$\mathfrak{T}^{\prime}(\alpha)$ composante alternative de $\mathfrak{T}_{\theta}(\alpha)$ (T/A).

$p_{\mathrm{s}}^{\prime}, p_{\mathrm{s}}$ nombre de paires de pôles statoriques bobinés d'excitation, de phase.

$p_{\mathrm{r}} \quad$ nombre de pôles saillants rotoriques.

$R_{0} \quad$ rayon moyen d'entrefer $(\mathrm{m})$.

$\theta$ position angulaire rotor/stator (rad.).

$\mathcal{U}_{\theta}(\alpha)$ différence de potentiel magnétique scalaire stator/ rotor (A).

$\mathcal{U}_{0}$ composante continue de $\mathcal{U}_{\theta}(\alpha)(\mathrm{A})$.

$\mathcal{U}^{\prime}(\alpha)$ composante alternative de $\mathcal{U}_{\theta}(\alpha)(\mathrm{A})$.

$W^{\prime}$ coénergie magnétique $(\mathrm{J})$.

$\left(^{*}\right)$ ERA du CNRS no 536.

\section{Introduction.}

Les possibilités nouvelles amenées par les alimentations de puissance à fréquence variable ont fait reconsidérer la machine à réluctance qui, pénalisée par une faible puissance massique à fréquence industrielle [1], n'était plus guère utilisée que pour des applications de petite puissance, principalement comme machine de positionnement.

Des recherches importantes concernant ces machines ont donc été entreprises durant ces dernières années, tout particulièrement sur le plan national dans le cadre du GRECO CNRS no 22 [2].

En dehors de leur qualité de robustesse bien connue, ces machines présentent la particularité de permettre un rapport élevé entre la fréquence des courants d'alimentation et la vitesse de synchronisme [3]. Fonctionnant comme un "réducteur magnéto-mécanique", la machine à réluctance à grand nombre de dents doit permettre ainsi d'obtenir un couple électromagnétique élevé.

Cependant cette structure possède une saillance peu élevée qui entraîne un faible facteur de puissance. Ceci implique donc un surdimensionnement du convertisseur statique par rapport à la puissance mécanique disponible [4]. 
Aussi afin de minimiser l'effet de la réaction d'induit une solution consiste-t-elle à greffer un enroulement d'excitation ou l'équivalent soit au rotor, soit au stator $[4,5]$.

Cette machine apparaît alors comme étant fondamentalement distincte de la machine à réluctance pure [6-8]. La synchronisation sur le couple d'excitation implique des structures particulières présentant, associées à des convertisseurs, des ondes de couple très différentes.

Afin de développer ces différents points, il est présenté dans un premier temps une étude théorique permettant de dégager les différentes structures des machines Vernier à excitation par courant continu statorique. Ensuite, découlant de cette étude, les auteurs font apparaître les diverses ondes de couple déduites du fonctionnement sur les seuls termes fondamentaux des grandeurs électriques et magnétiques.

Enfin, est réalisée une simulation de l'ensemble machine convertisseur, avec prise en compte de la saturation et moyennant l'hypothèse des commutations instantanées.

Une illustration de cette méthode est l'obtention d'une onde précise du couple instantané pour les différentes structures trouvées.

\section{Machines à réluctance Vernier excitées. Structures.}

1.1 EfFet réluctance. EfFet Vernier. - Le phénomène de réluctance variable appliqué aux machines électriques tournantes se traduit par une variation d'inductance des enroulements en fonction de la position du rotor. Ce dernier possède donc des pièces ferromagnétiques saillantes permettant ainsi de faire varier localement les réluctivités des trajets de flux.

A état magnétique constant une machine à réluctance non Vernier possède un couple proportionnel au nombre de pôles saillants $p_{\mathrm{r}}$. Une solution pour accroître le couple de cette machine consiste donc à augmenter $p_{\mathrm{r}}$. Cependant cette solution implique une multiplication de la polarité de phase dans le même rapport, ce qui aboutit à des densités de courant prohibitives le long de l'entrefer.

L'effet Vernier apporte une solution à ce problème en offrant la possibilité d'une augmentation du nombre de pôles saillants effectifs au rotor sans augmentation concomitante de la polarité des enroulements statoriques. Cet effet consiste en une double saillance statorique $\left(N_{\mathrm{s}}\right.$ dents) et rotorique $\left(N_{\mathrm{r}}\right.$ dents $), N_{\mathrm{r}}$ et $N_{\mathrm{s}}$ étant différents et généralement élevés (Fig. 1.1). Par effet de battement entre les deux pulsations spatiales $N_{\mathrm{s}}$ et $N_{\mathrm{r}}$ on obtient l'expression du fondamental de la perméance le long de l'entrefer :

$$
\mathfrak{T}_{\theta}(\alpha)=\mathfrak{T}_{0}+\mathfrak{T}_{1} \cos \left[\left(N_{\mathrm{r}}-N_{\mathrm{s}}\right) \alpha-N_{\mathrm{r}} \theta\right]
$$

soit en posant :

$$
\theta^{\prime}=\frac{N_{\mathrm{r}}}{N_{\mathrm{r}}-N_{\mathrm{s}}} \theta \text { et } \quad p_{\mathrm{r}}=N_{\mathrm{r}}-N_{\mathrm{s}}(1.1 .2)
$$
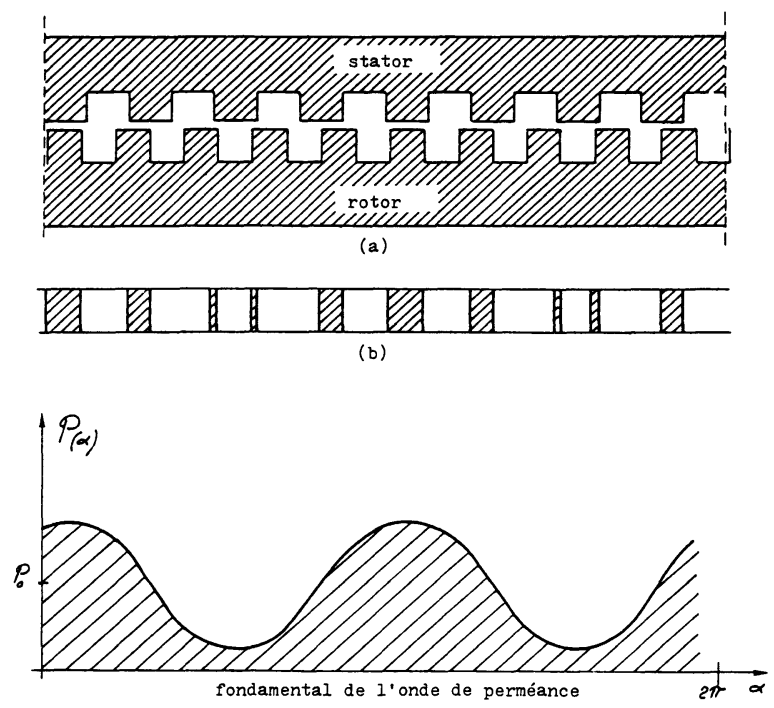

Fig. 1.1. - Denture d'une machine à réluctance Vernier.

[Reluctance machine teeth.]

$$
\mathfrak{T}_{\theta^{\prime}}(\alpha)=\mathfrak{T}_{0}+\mathfrak{T}_{1} \cos p_{\mathrm{r}}\left(\alpha-\theta^{\prime}\right) .
$$

La relation (3) montre que la machine $\operatorname{Vernier}\left(N_{\mathrm{s}}, N_{\mathrm{r}}\right)$ est équivalente à une machine non Vernier équivalente à $p_{\mathrm{r}}=\left(N_{\mathrm{r}}-N_{\mathrm{s}}\right)$ pôles saillants.

Ces deux machines développant bien entendu la même puissance, les vitesses de rotation sont dans le rapport $p_{\mathrm{r}} / N_{\mathrm{r}}$, les couples développés sont dans le rapport inverse.

On peut alors affirmer que la machine Vernier constitue un "moto-réducteur " électromagnétique susceptible de développer un fort couple à basse vitesse.

1.2 MÉTHOde D'ANALYSE. - Nous considérerons dans ce qui suit des machines Vernier polyphasées à excitation hétéropolaire par courant continu.

L'étude ayant pour but d'analyser la possibilité d'obtenir une conversion d'énergie magnétique-mécanique, seules les composantes spatiales des grandeurs électromagnétiques seront considérées.

La méthode proposée est basée sur l'interaction dans l'entrefer d'une onde de perméance $\mathfrak{T}_{\theta}(\alpha)$ et d'une onde spatiale de courant $\mathcal{U}_{\theta}(\alpha)$.

Les notations utilisées sont données à la figure 1.2.

$\mathcal{U}_{\theta}(\alpha)$ représentant le potentiel scalaire en un point $\alpha$ de l'entrefer a pour expression :

$$
u_{\theta}(\alpha)=R_{0} \int_{0}^{\alpha} \delta_{\text {is }}(\alpha) \mathrm{d} \alpha+u_{0}
$$

$\delta_{\text {is }}(\alpha)=$ densité linéique de courant le long du stator.

Quant à la fonction perméance, image de la géométrie de l'entrefer, nous supposerons qu'elle n'est pas déformée par la rotation du rotor, mais seulement translatée [4]. La fonction pourra s'écrire :

$$
\mathfrak{T}_{\theta}(\alpha)=\mathfrak{T}(\alpha-\theta) .
$$




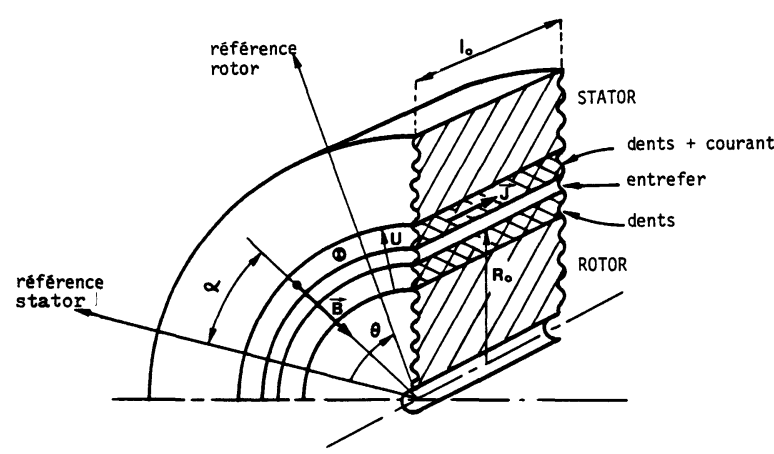

Fig. 1.2. - Machine à réluctance Vernier cylindrique.

[Cylindrical Vernier type reluctance machine.]

De plus elle sera prise indépendante de $\mathcal{U}_{\theta}(\alpha)$ ce qui implique un état non saturé de la machine.

L'interaction entre ces deux ondes va entraîner l'existence d'un couple électromagnétique dont l'expression, déduite des équations du champ après passage au potentiel scalaire, est :

$$
C=\frac{R_{0} l_{0}}{2} \frac{\mathrm{d}}{\mathrm{d} \theta}\left[\int_{0}^{2 \pi} \mathcal{U}_{\theta}^{2}(\alpha) \mathfrak{T}_{\theta}(\alpha) \mathrm{d} \alpha\right]
$$

1.3 PrincipauX TYPeS De STRUCTURES. - L'existence d'un couple d'excitation déduit de l'expression (1.2.3) va nous permettre de dégager les principales structures des machines Vernier excitées.

Nous allons pour cela développer en série de Fourier les deux fonctions $\mathcal{U}_{\theta}(\alpha)$ et $\mathfrak{T}_{\theta}(\alpha)$.

1.3.1 Développements en série de Fourier. - La fonction perméance peut alors s'écrire :

$$
\mathfrak{T}_{\theta}(\alpha)=\sum_{n=-\infty}^{+\infty} \beta_{n} \mathrm{e}^{j n\left(p_{\mathrm{r}} \alpha-\varepsilon N_{\mathrm{r}} \theta\right)}
$$

$\operatorname{avec} p_{\mathrm{r}}=\left|N_{\mathrm{r}}-N_{\mathrm{s}}\right|=\varepsilon^{\prime}\left(N_{\mathrm{r}}-N_{\mathrm{s}}\right)$

$$
\begin{array}{llll}
\varepsilon=\varepsilon^{\prime}=1 & \text { si } & N_{\mathrm{r}}>N_{\mathrm{s}} \\
\varepsilon=\varepsilon^{\prime}=-1 & \text { si } & N_{\mathrm{r}}<N_{\mathrm{s}} .
\end{array}
$$

L'existence de deux types de courant permet de développer $\mathcal{U}_{\theta}(\alpha)$ sous la forme :

$$
\mathcal{U}_{\theta}(\alpha)=\sum_{k=-\infty}^{+\infty} \gamma_{k} \mathrm{e}^{j k p_{s} \alpha}+\sum_{l=-\infty}^{+\infty} \gamma_{l}^{\prime} \mathrm{e}^{j l p_{s} \alpha}
$$

1.3.2 Potentiel rotorique. - L'expression (1.2.1) permet d'écrire :

$$
u_{\theta}(\alpha)=u_{0}(\theta)+u^{\prime}(\alpha) .
$$

$\mathcal{U}^{\prime}(\alpha)$ est à valeur moyenne nulle et $\mathcal{U}_{0}(\theta)$ représentera ce que nous appellerons le potentiel rotorique.

Nous aurons, compte tenu de (1.3.2) :

$$
u_{0}(\theta)=\gamma_{0}+\gamma_{0}^{\prime} .
$$

En écrivant de la même façon :

$$
\mathfrak{T}_{\theta}(\alpha)=\mathfrak{T}_{0}+\mathfrak{T}^{\prime}(\alpha-\theta)
$$

et en supposant qu'il n'existe aucun flux de type homopolaire dans la machine, nous aurons :

$u_{0}(\theta)=-\frac{1}{2 \mathfrak{T}_{0} \pi} \int_{0}^{2 \pi} u^{\prime}(\alpha) \mathfrak{T}^{\prime}(\alpha-\theta) \mathrm{d} \alpha$.

Soit, compte tenu de l'expression (1.3.2) :

$$
\begin{aligned}
\mathcal{U}_{0}(\theta)=-\frac{1}{\mathfrak{T}_{0}}\left[\sum_{n p \mathrm{r}+k p_{\mathrm{s}}=0} \gamma_{k} \beta_{n} \mathrm{e}^{-j \varepsilon n N_{\mathrm{r}} \theta}+\right. & \\
& \left.+\sum_{n p_{\mathrm{r}}+l p_{\mathrm{s}}=0} \gamma_{l}^{\prime} \beta_{n} \mathrm{e}^{-j \varepsilon N_{\mathrm{r}} n \theta}\right] .
\end{aligned}
$$

Ainsi de façon générale le potentiel rotorique est variable en fonction de la position du rotor.

1.3.3 Couple électromagnétique. - Compte tenu des expressions (1.3.3) et (1.3.5) le couple électromagnétique s'écrit :

$$
C=\frac{R_{0} l_{0}}{2} \frac{\mathrm{d}}{\mathrm{d} \theta}\left[\int_{0}^{2 \pi} \mathrm{u}^{\prime 2}(\alpha) \mathfrak{T}_{\theta}(\alpha) \mathrm{d} \alpha-2 \pi \mathfrak{T}_{0} \mathcal{u}_{0}^{2}(\theta)\right] .
$$

En introduisant les développements en série de $\mathcal{U}^{\prime}(\alpha)$ et $\mathfrak{T}_{\theta}(\alpha)$ nous obtenons l'expression suivante :

$$
\begin{aligned}
C= & -\varepsilon j \pi R_{0} l_{0} N_{\mathrm{r}}\left[\sum_{n p_{\mathrm{r}}+m p_{\mathrm{s}}=0} \sum_{k+l=m} n \gamma_{k} \gamma_{l} \beta_{n} \mathrm{e}^{-j \varepsilon n N_{\mathrm{r}} \theta}+\right. \\
& +C_{2}\left\{\sum_{n p_{\mathrm{r}}+m p_{\mathrm{s}}=0} \sum_{k+l=m} n \gamma_{k}^{\prime} \gamma_{l}^{\prime} \beta_{n} \mathrm{e}^{-j \varepsilon n N_{\mathrm{r}} \theta}\right. \\
& +C_{3}\left\{2 \sum_{n p_{\mathrm{r}}+m=0} \sum_{l p_{\mathrm{r}}^{\prime}+l p_{\mathrm{s}}^{\prime}=m} n \gamma_{k} \gamma_{l}^{\prime} \mathrm{e}^{-j \varepsilon n N_{\mathrm{r}} \theta}\right. \\
& +C_{4}\left\{-\pi R_{0} l_{0} \mathfrak{T}_{0} \frac{\mathrm{d}}{\mathrm{d} \theta}\left[\mathcal{U}_{0}^{2}(\theta)\right]\right]
\end{aligned}
$$

$n, k, l \neq 0$.

Ce couple est donc la superposition de quatre termes:

$C_{1}$ : couple de réluctance dû aux courants de phase.

$C_{2}$ : couple de réluctance dû au courant d'excitation.

$C_{3}$ : couple dû au couplage entre courants de phase et courant d'excitation.

$C_{4}$ : couple dû au potentiel rotorique.

1.3.4 Structures fonctionnant sur les termes fondamentaux de $\mathcal{U}_{\theta}(\alpha)$ et $\mathfrak{T}_{\theta}(\alpha)$. - Nous allons considérer que la machine excitée fonctionne sur l'interaction, crée par le fondamental de $\mathfrak{T}_{\theta}(\alpha)$, entre les fondamentaux des courants de phase et d'excitation. Le couple $C_{3}$ 
est différent de zéro si la relation :

$$
n p_{\mathrm{r}}+l p_{\mathrm{s}}^{\prime}+k p_{\mathrm{s}}=0
$$

est vérifiée pour $n= \pm 1, l= \pm 1, k= \pm 1$.

On en déduit alors l'expression générale liant les trois paramètres $p_{\mathrm{r}}, p_{\mathrm{s}}^{\prime}, p_{\mathrm{s}}$

$$
\text { avec } \quad \begin{aligned}
& p_{\mathrm{r}}=p_{\mathrm{s}}^{\prime}+\varepsilon_{0} p_{\mathrm{s}} \\
& \quad p_{\mathrm{r}}=\left|N_{\mathrm{r}}-N_{\mathrm{s}}\right| \\
& \varepsilon_{0}= \pm 1 .
\end{aligned}
$$

Etant donné les conditions d'existence des couples $C_{1}$ et $C_{2}$ de la relation (1.3.8) on en déduit l'existence de trois groupes de machines excitées fonctionnant sur les fondamentaux :

- Machines du 1 er groupe:

$$
\begin{aligned}
& p_{\mathrm{r}}=p_{\mathrm{s}}^{\prime}+\varepsilon_{0} p_{\mathrm{s}} \\
& p_{\mathrm{r}} \neq 2 p_{\mathrm{s}} \\
& p_{\mathrm{r}} \neq 2 p_{\mathrm{s}}^{\prime} .
\end{aligned}
$$

Ces machines sont caractérisées par l'existence d'un couple unique de type $C_{3}$.

- Machines du $2^{\mathrm{e}}$ groupe :

$$
\begin{aligned}
& p_{\mathrm{r}}=p_{\mathrm{s}}^{\prime}+\varepsilon_{0} p_{\mathrm{s}} \\
& p_{\mathrm{r}}=2 p_{\mathrm{s}} \\
& p_{\mathrm{r}} \neq 2 p_{\mathrm{s}}^{\prime} .
\end{aligned}
$$
$C_{1}$.

Ces machines possèdent un couple $C_{3}$ et un couple

- Machines du $3^{\mathrm{e}}$ groupe :

$$
\begin{aligned}
& p_{\mathrm{r}}=p_{\mathrm{s}}^{\prime}+\varepsilon_{0} p_{\mathrm{s}} \\
& p_{\mathrm{s}}^{\prime}=p_{\mathrm{s}} .
\end{aligned}
$$

Dans ce cas coexistent les trois couples $C_{1}, C_{2}, C_{3}$. Notons que pour les fondamentaux, $\mathcal{u}_{0}(\theta)$ est non nul si $p_{\mathrm{r}}=p_{\mathrm{s}}$ ou $p_{\mathrm{r}}=p_{\mathrm{s}}^{\prime}$.

Par conséquent, seules les machines du $1^{\text {er }}$ groupe sont susceptibles d'avoir un couple dû au potentiel rotorique. Ce couple est d'ailleurs nuisible au fonctionnement puisqu'il est toujours à valeur moyenne nulle.

\section{Etude simplifiée. Ondes de couple.}

Les structures étant maintenant définies, nous allons étudier les différentes formes d'ondes de couple présentées par chacune d'elles. Un classement vis-à-vis du critère stabilité en couple sera alors établi.

Pour cela nous avons choisi un échantillon représentatif de chaque type de machine, soit :

- machine du $1^{\text {er }}$ groupe $: p_{\mathrm{r}}=3 p_{\mathrm{s}} p_{\mathrm{s}}^{\prime}=2 p_{\mathrm{s}}$

- machine du $2^{\mathrm{e}}$ groupe $: p_{\mathrm{r}}=2 p_{\mathrm{s}} p_{\mathrm{s}}^{\prime}=3 p_{\mathrm{s}}$

- machine du $3^{\mathrm{e}}$ groupe $: p_{\mathrm{r}}=2 p_{\mathrm{s}} p_{\mathrm{s}}^{\prime}=p_{\mathrm{s}}$.
En outre, nous considérerons que ces machines sont triphasées et alimentées par convertisseurs statiques.

2.1 EXPRESSIONS ANALYTIQUES DES COUPLES. - Nous supposerons de plus que ces machines fonctionnent sur les fondamentaux des Fmm de phase et d'excitation, ainsi que de perméance

L'expression générale $(1.3 .8)$ du couple permet alors d'écrire :

- Machine du $1^{\mathrm{er}}$ groupe :

$C=-k \pi T_{1} N_{\mathrm{r}} v_{\mathrm{a}} v_{\mathrm{e}} \sin \left(N_{\mathrm{r}} \theta-\varphi-\varphi^{\prime}\right)$.

- Machine du 2 e groupe :

$$
\begin{array}{r}
C=2 k \pi N_{\mathrm{r}} \mathfrak{T}_{1}\left[\frac{v_{\mathrm{a}} v_{\mathrm{e}}}{2} \sin \left(N_{\mathrm{r}} \theta+\varphi-\varphi^{\prime}\right)-\right. \\
\left.-\frac{v_{\mathrm{a}}^{2}}{4} \sin \left(N_{\mathrm{r}} \theta-2 \varphi\right)\right] .
\end{array}
$$

- Machine du $3^{e}$ groupe :

$$
\begin{aligned}
C & =-2 k \pi N_{\mathrm{r}} \mathfrak{T}_{1}\left[\frac{v_{\mathrm{a}}^{2}}{4} \sin \left(N_{\mathrm{r}} \theta-2 \varphi\right)+\right. \\
& \left.+\frac{v_{\mathrm{a}}^{2}}{4} \sin \left(N_{\mathrm{r}} \theta-2 \varphi^{\prime}\right)+\frac{v_{\mathrm{a}} v_{\mathrm{e}}}{2} \sin \left(N_{\mathrm{r}} \theta-\varphi-\varphi^{\prime}\right)\right]
\end{aligned}
$$

avec : $\gamma_{1}=v_{\mathrm{a}}, \gamma_{1}^{\prime}=v_{\mathrm{e}}$

$\varphi=$ déphasage de la Fmm de phase par rapport à un axe de référence statorique,

$\varphi^{\prime}=$ déphasage de la Fmm d'excitation par rapport au même axe.

2.2 ONDES DYNAMIQUES. - La machine étant alimentée par convertisseur, son couple est déterminé pour chaque séquence de conduction (variation de l'angle $\varphi$ ). Nous supposerons que les commutations sont instantanées et que la fréquence d'alimentation est synchronisée sur le couple d'excitation.

Enfin, seule la forme des ondes nous intéressant, nous supposerons pour chaque machine que :

$$
2 k \pi N_{\mathrm{r}} \mathfrak{T}_{1}=1 \quad \varphi^{\prime}=0 .
$$

Les résultats obtenus (Fig. 2.1) montrent la très grande disproportion d'ondulation existant entre les trois machines. On constate que l'ondulation croît avec le nombre de couples de réluctance.

Ceci s'explique par le fait que les couples de réluctance sont alors des ondulations parasites à valeur moyenne nulle. En effet, la vitesse de synchronisation d'une machine à réluctance pure est le double de celle d'une machine excitée $[4,6]$.

$\mathrm{Du}$ point de vue stabilité, l'ordre de choix des machines est donc le suivant : $1^{\mathrm{er}}$ groupe, $2^{\mathrm{e}}$ groupe, $3^{\mathrm{e}}$ groupe. 

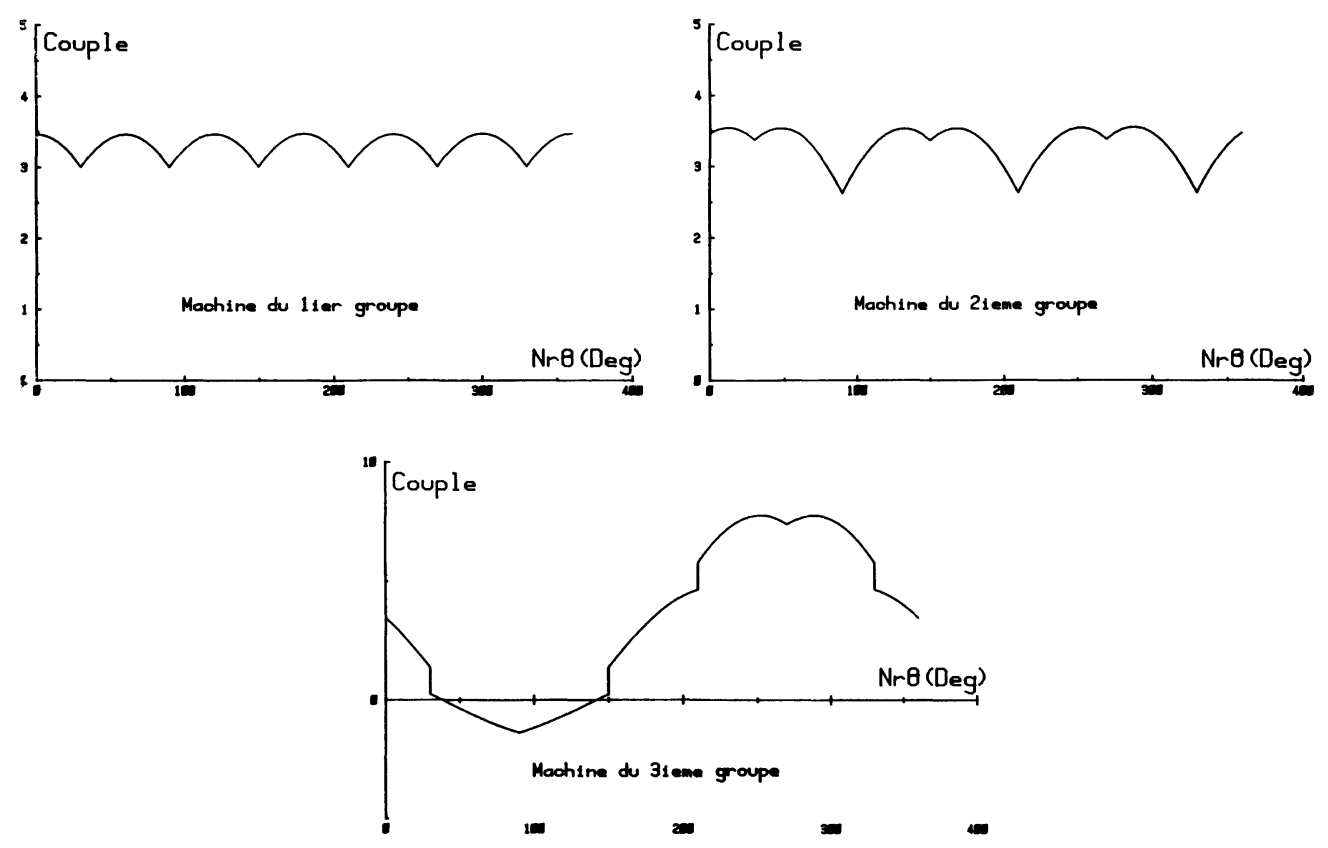

Fig. 2.1. - Ondulation de couple des 3 cas de machines excitées triphasées.

[Torque ripple for the three kinds of 3-phase excited reluctance machines.]

\section{Simulation des machines à reluctance excitées.}

L'étude précédente a été effectuée à partir de l'hypothèse d'interaction entre les seuls fondamentaux de force magnéto motrice et de perméance, ainsi que celle de linéarité du système.

Il est donc nécessaire pour obtenir des résultats quantitatifs précis d'avoir recours à une méthode de simulation beaucoup plus sophistiquée, faisant intervenir la structure magnétique réelle de la machine.

Les machines seront simulées compte tenu de leur alimentation soit, dans le cas présent, un système d'autopilotage avec commutateur de courant.

3.1 Modélisation. Principes. - Le modèle des circuits magnétiques équivalents s'adapte parfaitement aux machines à réluctance (Fig. 3.1). L'expression de la coénergie dans l'entrefer est alors :

$$
W^{\prime}=\sum_{\text {dents }} \int_{0}^{u_{j}} \varphi_{j} \mathrm{~d} u_{j}
$$

$\mathcal{u}_{j}$ : force magnétomotrice aux bornes de la dent $j$, $\varphi_{j}:$ flux à travers la dent $j$.

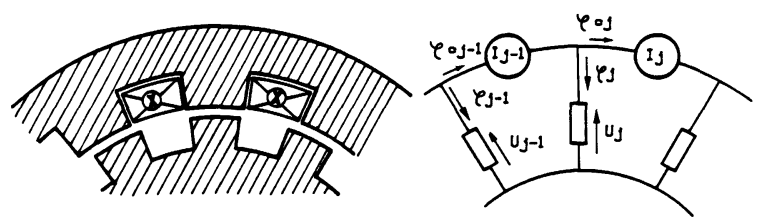

Fig. 3.1. - Circuit magnétique d'une machine à réluctance.

[Reluctance machine magnetic circuit.]
Le couple est déduit de $W^{\prime}$ par dérivation par rapport à l'angle de rotation du rotor.

Quant aux flux dans les enroulements, ils sont calculés à partir des flux de culasses $\varphi_{c j}$, la relation liant ces derniers étant :

$$
\varphi_{\mathrm{cj}}=\varphi_{\mathrm{cj}-1}-\varphi_{j}
$$

Le calcul de $\varphi_{\mathrm{c} j}\left(\mathcal{U}_{j}\right)$ est réalisé moyennant deux hypothèses :

- hypothèse de non-saturation de la culasse,

- hypothèse de variation sinusoïdale du flux dentaire local soit :

$$
\varphi_{j}=\frac{\varphi_{\mathrm{d} j}+\varphi_{\mathrm{q} j}}{2}+\frac{\varphi_{\mathrm{d} j}-\varphi_{\mathrm{q} j}}{2} \cos N_{\mathrm{r}} \theta_{j}
$$

$\varphi_{\mathrm{d} j}$ : flux de la dent $j$ en position directe avec une dent rotorique

$\varphi_{\mathrm{q} j}$ : flux de la dent $j$ en position de quadrature avec 、 une dent rotorique

$\theta_{j}$ : angle entre la dent $j$ et l'axe statorique.

Les flux $\varphi_{d}(U)$ et $\varphi_{q}(U)$ sont déterminés par calcul de champ à l'aide du programme DIFIMEDI (Fig. 3.2).

Ces deux courbes seront donc les caractéristiques de la denture utilisée. La prise en compte de la saturation se fait également à leur niveau.

3.2 EXPloitation DU PROGRAMME DE SIMULATION. ONDES DE COUPLE. - Afin d'établir une comparaison entre l'analyse simplifiée et la simulation, les trois structures échantillons des trois groupes ont été étudiées. 


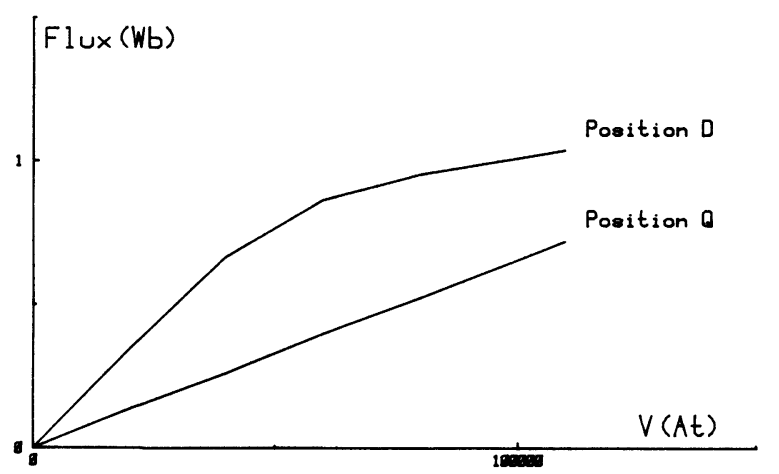

Fig. 3.2. - Flux dentaires en position directe en quadrature. [Maximum and minimum tooth flux.]

Les résultats concernant l'évolution du couple en fonction de la position rotorique (Fig. 3.3) sont très semblables aux précédents en ce qui concerne la structure du $2^{\mathrm{e}}$ groupe. Quant aux deux autres, elles diffèrent très nettement, ceci s'expliquant par l'absence de prise en compte de la dissymétrie de bobinage de ces machines lors de l'étude analytique. La relative stabilité des structures symétriques du deuxième groupe apparaît donc ici très nettement.

Le rôle dissymétrique des enroulements d'excitation vis-à-vis des enroulements de phase pour des machines $\mathrm{du} 1^{\mathrm{er}}$ et $3^{\mathrm{e}}$ groupe entraîne des sauts de couple très nets dus aux commutations.

Laissant de côté la machine du $3^{\mathrm{e}}$ groupe, le caractère symétrique semble donc avantager très nettement la structure du $2^{\mathrm{e}}$ groupe vis-à-vis de celle du $1^{\mathrm{er}}$ groupe et ceci malgré le fait que cette dernière ne présente qu'un couple d'excitation, donc théoriquement et $a$ priori aucune ondulation parasite.
Au vu de ces constatations, il apparaît que la structure idéale serait une machine symétrique du $1^{\mathrm{er}}$ groupe, qui allie symétrie et unicité de couple $\left(p_{\mathrm{r}}=3 p_{\mathrm{s}}, p_{\mathrm{s}}^{\prime}=3 p_{\mathrm{s}}\right)$.

La figure 3.4 montre les résultats de simulation de cette machine ainsi que ceux de la machine symétrique du $2^{\mathrm{e}}$ groupe.

On peut constater à point de fonctionnement identique (même induction maximale) une ondulation minimale pour la machine du $1^{\mathrm{er}}$ groupe, mais également un couple moyen plus faible que celui de la machine du $2^{\mathrm{e}}$ groupe.

Il faut également noter que bien qu'appartenant au $1^{\text {er }}$ groupe la machine simulée possède néanmoins des couples de réluctances parasites provenant des harmoniques de Fmm de rang supérieur à 1 .

En effet, ces derniers induisent des décalages non symétriques des arches de couples, provoquant ainsi une ondulation supplémentaire.

\section{Conclusion.}

Dans une première partie, une étude analytique des structures excitées a été mise en œuvre. Les fonctions perméance et potentiel magnétique scalaire ont permis de caractériser une structure générale et de donner une expression simple du transfert électromécanique d'énergie.

Le critère d'existence d'un couple d'excitation associé à la transformation de Fourier des deux fonctions permet alors de dégager trois groupes de structures : les machines à couple d'excitation seul, les machines à couple d'excitation et à couple de réluc-
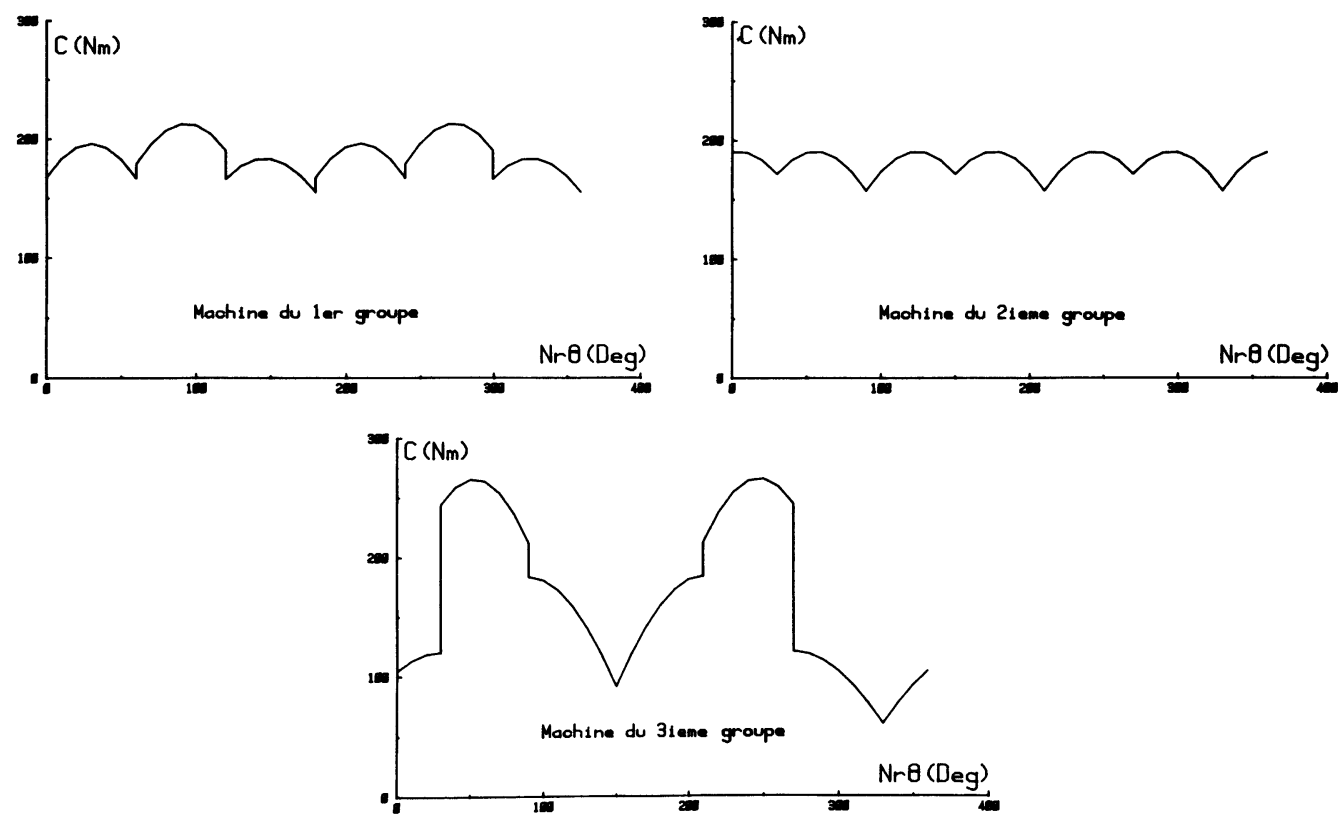

Fig. 3.3. - Couples dynamiques des 3 cas de machines excitées triphasées.

[Instantaneous torque for the three kinds of 3-phase excited reluctance machines.] 


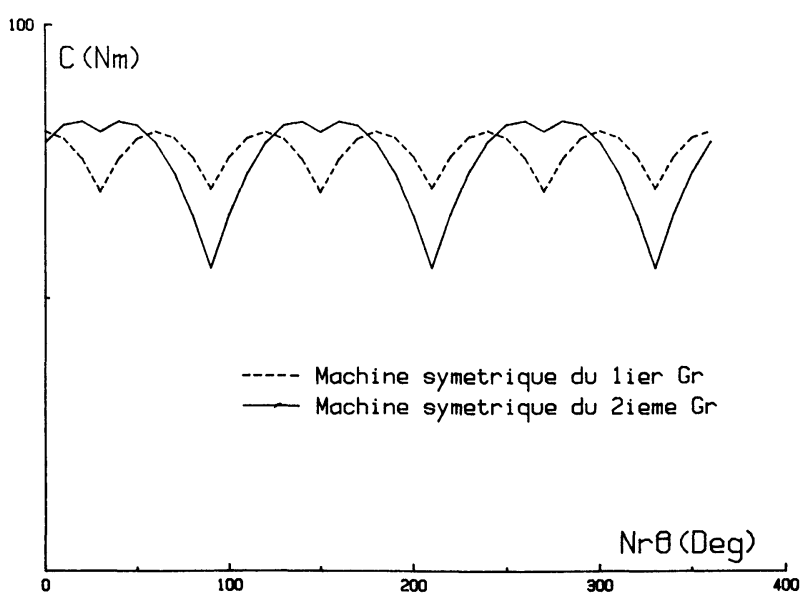

Fig. 3.4. - Couples dynamiques des machines symétriques triphasées du $1^{\text {er }}$ et $2^{\mathrm{e}}$ groupe.

[Instantaneous torque for symetrical 3-phase machines of 1 st and 2 nd type.]

tance sur les enroulements de phase, enfin les machines à couple d'excitation et à couple de réluctance sur les deux types d'enroulements.
La deuxième partie a été consacrée à l'évolution instantanée du couple d'une machine associée à un convertisseur. Une première approche a été réalisée à partir de l'étude de la première partie et a permis de montrer l'ondulation minimale de couple des machines du 1 er groupe.

Enfin, pour prendre en compte la géométrie complexe des machines Vernier ainsi que les formes d'ondes réelles, une simulation des trois types de structures a été réalisée et a confirmé, avec plus de précision, la structure optimale des machines symétriques du $1^{\mathrm{er}}$ groupe, compte tenu, bien sûr, du critère stabilité en couple.

Il faut cependant remarquer que la symétrie de bobinage implique pour la machine du $1^{\mathrm{er}}$ groupe un nombre d'encoches courant par pôle et par phase toujours supérieur à celui d'une machine du $2^{\mathrm{e}}$ groupe. Ceci se traduit dans tous les cas par un couple développé plus faible pour la machine symétrique du $1^{\text {er }}$ groupe.

Enfin, il faut noter que pour un nombre de phases différent de trois une machine symétrique sera toujours du $1^{\text {er }}$ groupe et possédera par conséquent la plus grande stabilité en couple.

\section{Bibliographie}

[1] Carpenter, C. J., Magnetic equivalent circuits, Proc. IEE 115 (1965).

[2] Rioux, C., Faucher, J., Lucidarme, J., SARgos, F. M., Adaptation des machines à réluctance variable aux systèmes d'entraînements. Proc. Colloque Européen : Au carrefour de la force motrice. Paris, 10/11 décembre 1981.

[3] LEE, C. H., The theory and design of a very slow speed reluctance motor, ATEE General Meeting, Chicago, octobre 1959.

[4] FAUCHER, J., Contribution à l'étude des machines à réluctance variable à commutation électronique, Thèse de Docteur ès Sciences, INP Toulouse, juin 1981.
[5] BANON, L., Contribution à l'optimisation des machines à réluctance variable de type Vernier à commutation électronique. Thèse de Docteur Ingénieur, INP Toulouse, novembre 1981.

[6] Sargos, F. M., Etude théorique des performances des machines à réluctance variable, Thèse de Docteur ès Sciences, INPL Nancy, mars 1981.

[7] Rapport GRECO 1981 « Machines électriques à hautes performances $»$.

[8] ZEISSER, J., Contribution à l'étude des machines à réluctance à plots dentés, Thèse de Docteur Ingénieur, INPL Nancy, janvier 1980. 